\title{
MANGANESE DISTRIBUTION IN CdMnTeSe CRYSTALS. EXAFS DATA ANALYSIS*
}

\author{
A. Kisiel, J. Eażewski ${ }^{\dagger}$, M. Zimnal-Starnawska \\ Institute of Physics, Jagiellonian University, Reymonta 4, 30-059 Kraków, Poland
}

E. Burattini

Laboratori Nazionali di Frascati, INFN, via E. Fermi 40, 00044 Frascati, Italy

\author{
AND A. MYCIELSKI
}

Institute of Physics, Polish Academy of Sciences

Al. Lotników 32/46, 02-668 Warszawa, Poland

The extended X-ray absorption fine structure (EXAFS) technique has been used to investigate the first shell coordination in zinc-blende quaternary $\mathrm{Cd}_{1-x} \mathrm{Mn}_{x} \mathrm{Te}_{1-y} \mathrm{Se}_{y}(0.0 \leq x \leq 0.15 ; 0.0 \leq y \leq 0.20)$ alloys. The experimental data have been collected with the use of synchrotron radiation. All the nearest-neighbour distances have been found as linearly dependent on $\mathrm{Se}$ and $\mathrm{Mn}$ concentration. For each composition a consistent deviation from full random distribution of $\mathrm{Mn}$ atoms around Se and Te atoms was observed. Possible reasons of preferential $\mathrm{Mn}-\mathrm{Se}$ over $\mathrm{Mn}-\mathrm{Te}$ pairing were discussed.

PACS numbers: $61.10 .-\mathrm{i}, 61.72 . \mathrm{Vv}, 75.50 . \mathrm{Pp}$

In tetrahedrally coordinated III-V and II-VI semiconducting compounds there exist two sublattices occupied respectively by anions and cations. In $A_{1-x} B_{x} \mathrm{C}$ and $\mathrm{AC}_{1-y} \mathrm{D}_{y}$ ternary or $\mathrm{A}_{1-x} \mathrm{~B}_{x} \mathrm{C}_{1-y} \mathrm{D}_{y}$ quaternary compounds a basic question concerns a random or non-random substitution of the $A$ by $B$ and $C$ by $D$ ions in respective sublattices. The first answer to this question has been obtained from infrared phonon mode analysis for $\mathrm{GaP}_{1-y} \mathrm{As}_{y}$ [1], $\mathrm{CdS}_{1-y} \mathrm{Se}_{y}$ [2], $\mathrm{Cd}_{1-x} \mathrm{Hg}_{x} \mathrm{Te}$ [3] and $\mathrm{CdTe}_{1-y} \mathrm{Se}_{y}$ [4] ternary alloys. In all these experiments an additional feature in the infrared reflectivity spectrum has been observed and interpreted as a result of next nearest neighbours clustering [1-4]. The information concerning the partial clustering of ternary compounds have been also obtained from nuclear magnetic resonance in CdHgTe [5] and recently, in extended X-ray absorption fine structure (EXAFS) analysis for diluted magnetic semiconductors $\mathrm{ZnMnSe} \mathrm{[6],} \mathrm{as} \mathrm{well}$ as for non-magnetic quaternary $\mathrm{In}_{1-x} \mathrm{Ga}_{x} \mathrm{Sb}_{1-y} \mathrm{As}_{y}$ alloys [7]. EXAFS analysis for $\mathrm{ZnMnSe}[6]$ shows that $\mathrm{Zn}-\mathrm{Mn}$ coordination number is slightly larger, and $\mathrm{Zn}-\mathrm{Zn}$

* Supported by the Committee for Scientific Research grant number PB-1147/P3/94/06.

I Also at the Institute of Nuclear Physics, Radzikowskiego 152, 31-342 Kraków, Poland. 
slightly smaller than predicted by virtual crystal approximation (VCA). Pong et al. [6] explained this deviation from randomness, mainly as an influence of $\mathrm{Mn}-\mathrm{Mn}$ superexchange interaction in $\mathrm{ZnMnSe}$. In quaternary alloys, atomic correlations are expected to be larger than for ternaries because nearest-neighbours preferences are possible. Islam and Bunker [7] found in InGaSbAs quaternary compounds a preference of $\mathrm{Ga}-\mathrm{As}$ pairs over In-As pairs, which they argue for the sequences in the chemical potentials of binaries and the differences of the electronic bonding and antibonding energy levels. Unfortunately, minimization of the strain energy in InGaSbAs leads to opposite preferences, i.e. the preference $\mathrm{Ga}-\mathrm{Sb}$ and $\mathrm{In}-\mathrm{As}$ pairs.

The aim of this paper is to analyze discrepancies in random distribution of quaternary $\mathrm{Cd}_{1-x} \mathrm{Mn}_{x} \mathrm{Te}_{1-y} \mathrm{Se}_{y}$ diluted magnetic semiconductors. Experimental EXAFS measurements have been carried out with the use of synchrotron radiation at the Adone wiggler facility in Frascati applying the $\mathrm{Si}(111)$ and $\mathrm{Si}(220)$ channel-cut crystal monochromators [8]. The original samples were high purity monocrystalline binary CdTe, CdSe, MnTe and MnSe standard compounds, ternaries CdMnTe (also examined before in [9]) and $\mathrm{Cd}_{0.75} \mathrm{Mn}_{0.25} \mathrm{Se}$, as well as $\mathrm{Cd}_{1-x} \mathrm{Mn}_{x} \mathrm{Te}_{1-y} \mathrm{Se}_{y}(x=0.00,0.05,0.10,0.15 ; y=0.00,0.10,0.20)$ quaternary compounds produced by Bridgman method. The binary compounds crystallize in different structures: CdTe in zinc-blende structure, $\mathrm{MnSe}$ in $\mathrm{NaCl}$ struc-

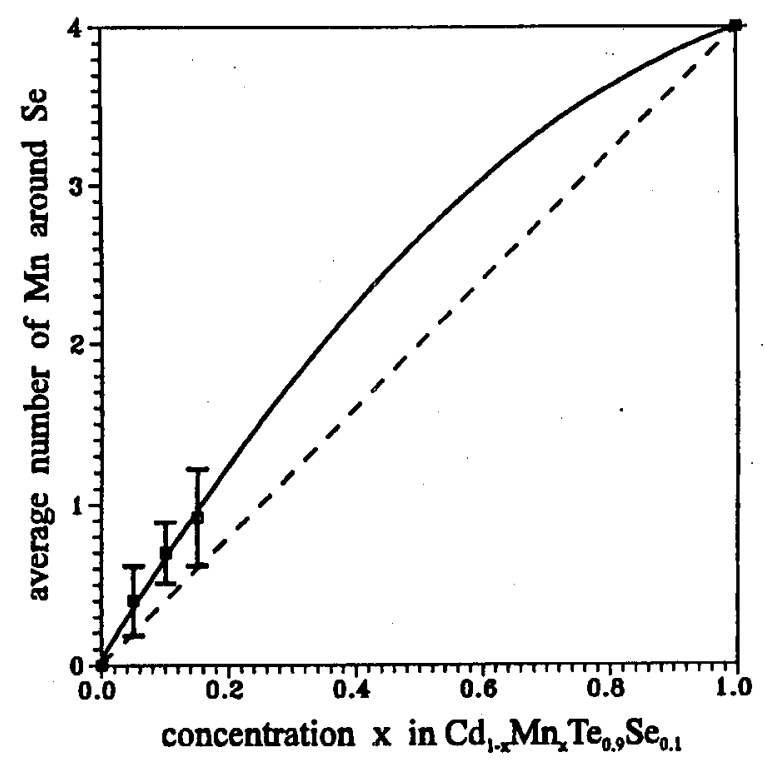

Fig. 1. Mn-Se coordination as a function of Mn concentration in $\mathrm{Cd}_{1-x} \mathrm{Mn}_{x} \mathrm{Te}_{0.9} \mathrm{Se}_{0.1}$ from Se $K$-edge. The solid line (square function) was fitted to experimental points (open squares) for $5 \%, 10 \%, 15 \%$ of $\mathrm{Mn}$ with $10 \%$ of Se as well as to two border points for ternaries $\mathrm{CdTe}_{0.9} \mathrm{Se}_{0.1}$ and hypothetical zinc-blende $\mathrm{MnTe}_{0.9} \mathrm{Se}_{0.1}$ compounds. The dashed line indicates the full random distribution of dopants. 
ture, MnTe and CdSe in hexagonal NiAs and in wurtzite structures, respectively. $\mathrm{Cd}_{1-x} \mathrm{Mn}_{x} \mathrm{Te}_{1-y} \mathrm{Se}_{y}$ compounds crystallize in well defined monophase zinc-blende structure, according to its phase diagram [10], only for $x \leq 0.2$ when $y \leq 0.2$ and $x \leq 0.7$ when $y \leq 0.05$. All compositions of studied samples were derived from this monophase region. The contribution of each edge to the absorption coeffcient has been isolated by extrapolating the pre-edge region to higher energies by a Victoreen-like fit and by subtracting the fitted curves from the remaining experimental spectrum [11]. EXAFS data analysis for $M n$ and Se $K$-edges was done applying the well known data reduction procedure described elsewhere [12]. In this paper we were mainly interested in the first neighbours distribution. The one-shell parameters $(N, R$ and $\sigma)$ were obtained by non-linear least squares fitting. Figure 1 shows the $\mathrm{Mn}-\mathrm{Se}$ coordination number as a function of $\mathrm{Mn}$ composition taken from Se $K$-edge. The consistent deviation from random distribution (represented by dashed line in Fig. 1) is visible. These results are confirmed by those obtained from $\mathrm{Mn} K$-edge (Mn neighbourhood). The preferences observed in quaternary CdMnTeSe could be explained, similarly to Pong et al. suggestions, by the predominant influence of the superexchange interaction which is larger in $\mathrm{MnSe}$ and $\mathrm{CdMnSe}$ than in $\mathrm{MnTe}$ and CdMnTe, respectively [13]. As the deviation from randomness in non-magnetic InGaSbAs has been also discovered [7], therefore, in CdMnTeSe apart from the magnetic interactions the structural reasons of the preferences should be additionally taken into consideration.

\section{References}

[1] H.W. Verleur, A.S. Barker Jr., Phys. Rev. 149, 715 (1966).

[2] H.W. Verleur, A.S. Barker Jr., Phys. Rev. 155, 750 (1967).

[3] S.P. Kozyrev, L.K. Vodopyanov, R. Triboulet, Solid State Commun. 45, 383 (1983).

[4] S. Perkowitz, L.S. Kim, P. Becla, Phys. Rev. B 43, 6598 (1991).

[5] D. Zamir, K. Beshah, P. Becla, P.A. Wolff, R.G. Griffin, D. Zax, S. Vega, N. Yellin, J. Vac. Sci. Technol. A 6, 2612 (1988).

[6] W.-F. Pong, R.A. Mayanovic, B.A. Bunker, J.K. Furdyna, U. Debska, Phys. Rev. $B$ 41, 8440 (1990).

[7] S.M. Islam, B.A. Bunker, Phys. Lett. A 156, 247 (1991).

[8] E. Burattini, E. Bernieri, A. Balerna, C. Mencuccini, R. Rinzivillo, G. Dalba, P. Fornasini, Nucl. Instrum. Methods Phys. Res. A 246, 125 (1986).

[9] A. Balzarotti, N. Motta, A. Kisiel, M. Zimnal-Starnawska, M.T. Czyżyk, M. Podgónny, Phys. Rev. B 31, 7526 (1985).

[10] S. Chehab, G. Lamarche, A. Manoogian, J.C. Wulley, J. Magn. Magn. Mater. 59, 105 (1986).

[11] B.K. Teo, EXAFS: Basic Principles and Data Analysis, Springer Verlag, Berlin 1986.

[12] F.W. Lytle, D.E. Sayers, E.A. Stern, Physica B 158, 701 (1989).

[13] A. Lewicki, J. Spałek, J.K. Furdyna, R.R. Gałązka, J. Magn. Magn. Mater. 54-57, 1221 (1986). 